\title{
Thrombolysis Is an Effective and Safe Therapy in Stuck Mitral Valves With Delayed Presentation as Well as Hemodynamically Unstable Patients: A Single Centre Study
}

\author{
Arun Shivajirao Bade ${ }^{a}$, Shakil Sattar Ahmed Shaikha, b, Hemant Khemania, \\ Gurkirat Singha $^{\mathrm{a}}$, Narender Omprakash Bansal ${ }^{\mathrm{a}}$
}

\begin{abstract}
Background: Thrombosis is a complication of prosthetic valves on oral anticoagulants which is associated with significant morbidity and mortality. A re-operation carries a substantial risk, with mortality rate from $10 \%$ to $15 \%$ in selected series, which may be 2 - or 3 -folds higher in critically ill patients. This study conducted in a tertiary care cardiology unit aimed to evaluate the effectiveness and safety of thrombolytic therapy in stuck mitral bileaflet heart valves.
\end{abstract}

Methods: As a prospective observational study, clinical symptoms and fluoroscopy were the mainstay in diagnosis of stuck mitral valve. Gradient across the valve by transthoracic echocardiography was used to monitor the therapy every $6 \mathrm{~h}$. Fall of mean gradient more than $50 \%$ was considered as successful thrombolysis. And final results were again checked by fluoroscopy with documentation of improved leaflet movement.

Results: Totally we studied 34 patients. Patients receiving thrombolytic therapy with streptokinase achieved an overall $91.2 \%$ freedom from a repeat operation or major complications, a large subcutaneous hematoma occurred in one $(2.9 \%)$, reoperation required in two due to failure of treatment $(5.9 \%)$, allergic reaction in one $(2.9 \%)$, one patient developed transient neurologic dysfunction (2.9\%) and one patient died during therapy due to refractory cardiogenic $\operatorname{shock}(2.9 \%)$. All patients including those with delayed presentation ( $>14$ days) and hemodynamically unstable patients had good results similar to those who presented within 14 days and hemodynamically stable. Mortality was higher in unstable patients and reoperation was higher with delayed presentation.

Conclusions: Thrombolysis with streptokinase is highly successful and safe therapy in hemodynamically stable as well as unstable patients, or those with early or delayed presentation with stuck bileaflet

Manuscript submitted March 27, 2018, accepted April 17, 2018

aDepartment of Cardiology, Grant Medical College and Sir JJ Group of Hospitals, Mumbai India

${ }^{\mathrm{b} C}$ Corresponding Author: Shakil Sattar Ahmed Shaikh, 201, Ayesha Manzil, A Wing Sunrise Galaxy Housing Society, Dr. Ambedkar Road, Kalyan (west), Maharashtra, India. Email: drshakilsshaikh@rediffmail.com

doi: https://doi.org/10.14740/cr708w mitral valves, especially in centers where round the clock cardiothoracic surgery backup is not available.

Keywords: Stuck mitral valves; Thrombolysis; Prosthetic valve

\section{Introduction}

Presently bileaflet valves are the mechanical valves of choice in prosthetic mitral valve implantation. Data focusing on these valve models with respect to valve thrombosis are emerging only in the last decade [1-10]. This study enlightens some aspects unique to thrombosed bileaflet valves and their implications on the feasibility and safety of thrombolysis. Different prosthetic valve models may vary in their propensity for thrombosis, the relative interrelations and proportion of ingrowth tissue and thrombus, the amount of thrombus needed to disturb leaflet motion and the vulnerable zones within the valve. Bileaflet valves are of special interest because their delicate mechanism may lead more easily to leaflet immobilization, even with a relatively small clot. On the other hand, if the offending clot is minor, thrombolysis may be easier and safer. Therefore, we sought to explore the role of thrombolysis in stuck bileaflet mitral valves based on our own experience and a literature review.

\section{Methods}

As a prospective observational study, we totally studied 34 patients admitted in our tertiary care intensive care unit from January 2017 to December 2017. Clinical symptoms profile of patient and fluoroscopy were the mainstay in diagnosis and evaluation of leaflet mobility in cases of stuck mitral valve. The desired acquisition angle was obtained in all cases. Transthoracic echocardiogram (TTE) was performed in all patients, using all standard views. The findings in TTE raised the suspicion of stuck valve and mandated further studies were increased mean diastolic transvalvular gradients $(>6 \mathrm{~mm} \mathrm{Hg})$ and failure to achieve at least one view demonstrating movement of both leaflets. Transesophageal echocardiography (TEE) studies were performed in all patients before throm- 


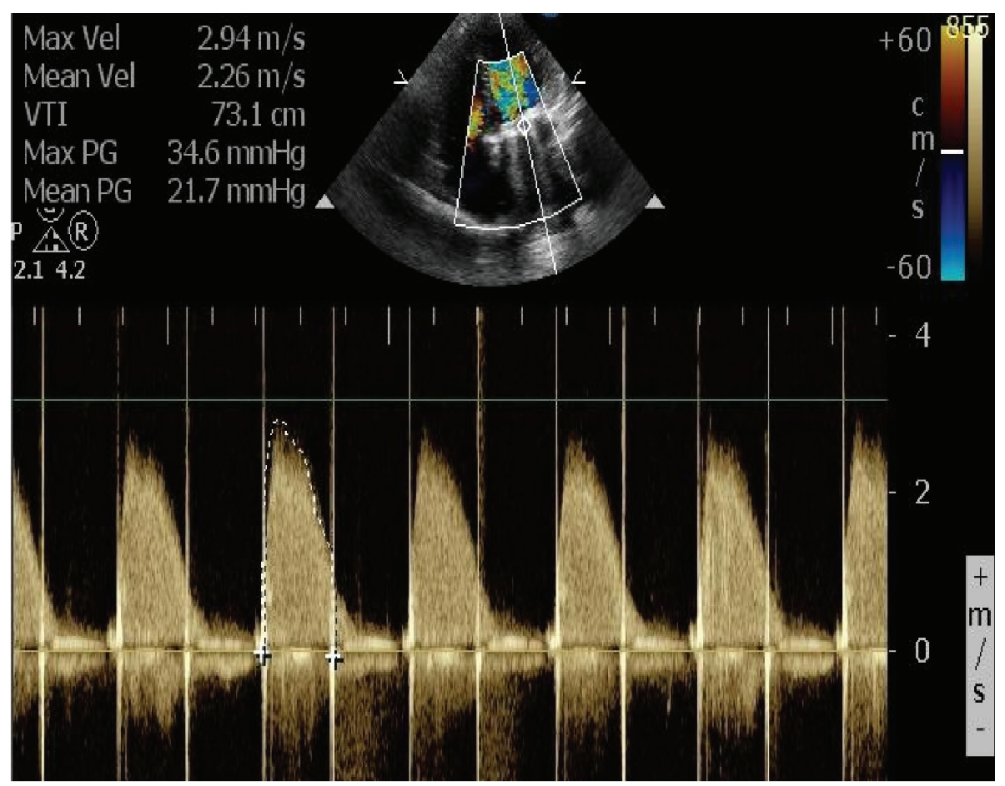

Figure 1. TTE image before thrombolysis shows mean gradient of $21.7 \mathrm{~mm} \mathrm{Hg}$ across stuck prosthetic mitral valve.

bolysis except those who were in frank pulmonary edema, to exclude a mobile large $(8 \mathrm{~mm})$ thrombus, which was considered a contraindication for thrombolysis in our institute [9]. Details of valve implantation, its model, size and position were gathered from the patient's record. Patients' symptoms were recorded, including symptom duration, heart failure (expressed as New York Heart Association (NYHA) class), embolic phenomena (peripheral, coronary, cerebral), palpitations and syncope.

\section{Treatment protocol}

Intravenous thrombolysis was given, using streptokinase (STK). Streptokinase was usually given as a 30 -min loading dose of 150,000 to $250,000 \mathrm{U}$, followed by $100,000 \mathrm{U} / \mathrm{h}$. STK was administered until valve reopening or for at least $24 \mathrm{~h}$. Intravenous heparin infusion was started after termination of thrombolysis. After stabilization concomitantly warfarin was administered to achieve target $(2.5$ - 3.5) international normalised ratio (INR) levels. Heparin was discontinued after two therapeutic INR levels were achieved, $24 \mathrm{~h}$ apart. In addition, all patients were started on aspirin (100 mg/day).

\section{Evaluation of treatment outcome}

Each patient underwent TTE evaluation every $6 \mathrm{~h}$ from the start of thrombolytic treatment and on a daily basis thereafter if full valve reopening was not achieved. Clues for valve reopening were clear-cut evidence of movement of both leaflets in a parallel fashion and a substantial (50\%) decrease in transvalvular gradients (Fig. 1, 2). Confirmation of valve reopening was achieved by fluoroscopy, which was performed in each patient within $24 \mathrm{~h}$ after completion of thrombolytic course.

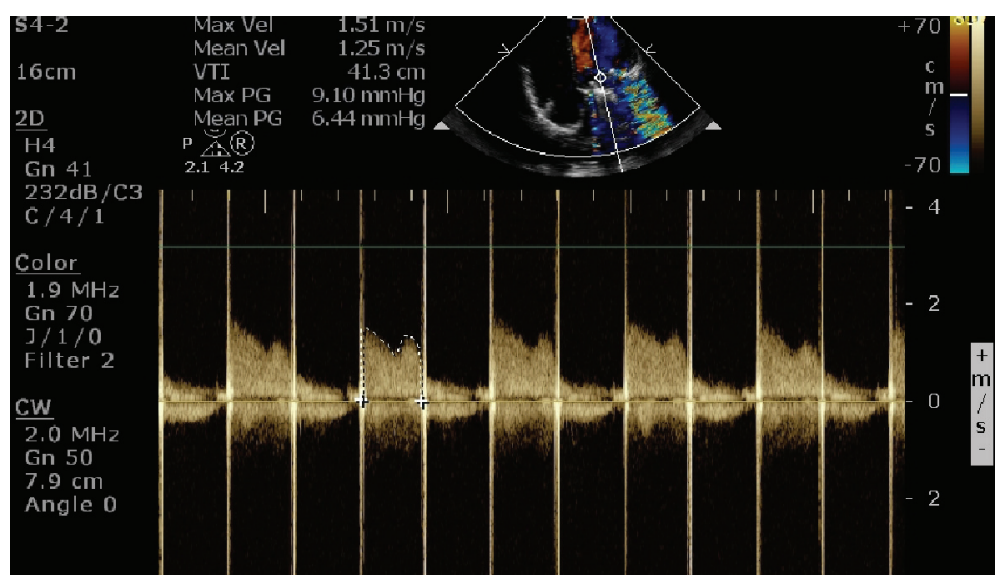

Figure 2. TTE image at $24 \mathrm{~h}$ of thrombolysis shows significant fall in mean gradient to $6.44 \mathrm{~mm} \mathrm{Hg}$. 
Table 2. Overall Results of Thrombolysis in 34 Patients With Stuck Mitral Valves

\begin{tabular}{|c|c|}
\hline Parameters & Results \\
\hline Clinical success rate & $31(91.2 \%)$ \\
\hline Re-operation required & $2(5.9 \%)$ \\
\hline Mortality & $1(2.9 \%)$ \\
\hline \multicolumn{2}{|l|}{ Complications } \\
\hline Large subcutaneous hematoma & $1(2.9 \%)$ \\
\hline Neurological deficit (TIA) & $1(2.9 \%)$ \\
\hline Allergic reaction & $1(2.9 \%)$ \\
\hline
\end{tabular}

\section{Complications}

Major bleeding was defined as any bleeding that necessitated blood transfusion or was associated with a decrease of $3 \mathrm{~g} / \mathrm{dL}$ or more in blood hemoglobin concentration. Otherwise, bleeding was termed minor. A careful neurologic examination and brain computed tomography were performed in any patient who had neurologic complaints during or after the thrombolytic infusion. Fever with chills and generalized rash were considered indicative of drug allergy, unless otherwise explained.

\section{Follow-up after discharge}

Each patient was discharged with detailed instructions about the importance of anticoagulation regimen, common factors interacting with the warfarin efficacy and maintenance of INR in therapeutic window of 2.5 - 3.5; also explained about signs and symptoms of stuck valve. Follow-up in the cardiology clinic included patient history, physical examination and echocardiography at 1 months followed by 3 monthly after discharge and biannually thereafter. Fluoroscopy was done in cases to rule out repeated restriction of leaflet motion whenever TTE showed high transvalvular gradients or leaflet motion seemed imperfect.

\section{Results}

we studied total 34 patients; 10 males $(29.4 \%)$ and 24 females $(70.6 \%) ; 24$ were $<40$ years $(70.6 \%)$ and 10 were $>40$ years $(29.4 \%)$ who experienced one or more episodes of stuck valve over a 12-months period and received thrombolysis with streptokinase. A total of 27 patients (79.4\%) were presented within 14 days, and seven patients (20.6\%) after more than 14 days of symptoms. Functional class at initial episode was NYHA I-II in 12 patients $(35.3 \%)$ and NYHA III-IV in 22 patients $(64.7 \%)$, 25 patients $(73.5 \%)$ were hemodynamically stable and nine patients $(26.5 \%)$ were hemodynamically unstable (i.e. blood pressure (BP) $<90 \mathrm{mmHg}$ systolic) on presentation(Table 1). During study period, patients receiving thrombolytic therapy with streptokinase achieved an overall $91.2 \%$ freedom from a repeat operation or major complications, a large subcutaneous
Table 1. Patients' Characteristics

\begin{tabular}{ll}
\hline Patients characteristics & Result \\
\hline $\begin{array}{l}\text { Number of patients } \\
\text { Age }\end{array}$ & 34 \\
$\quad$ Less than 40 years & $24(70.6 \%)$ \\
$\quad$ More than 40 years & $10(29.4 \%)$ \\
Gender & \\
$\quad$ Male & $10(29.4 \%)$ \\
$\quad$ Female & $24(70.6 \%)$ \\
Symptoms duration & $27(79.4 \%)$ \\
$\quad$ Less than 14 days & $7(20.6 \%)$ \\
$\quad$ More than 14 days & $12(38.3 \%)$ \\
Initial functional class & $22(64.7 \%)$ \\
I - II & \\
III - IV & $25(73.5 \%)$ \\
Hemodynamically & $09(26.5 \%$ \\
Stable &
\end{tabular}

hematoma occurred in one $(2.9 \%)$, reoperation required in two $(5.9 \%)$, allergic reaction in one $(2.9 \%)$, one patient developed transient neurologic dysfunction $(2.9 \%)$ and one patient died during therapy due to refractory cardiogenic shock $(2.9 \%)$ (Table 2). All patients including those with delayed presentation ( $>$ 14 days) and hemodynamically unstable patients had good results similar to those who presented within 14 days and hemodynamically stable patients. Mortality was higher in unstable patients and reoperation was higher with delayed presentation.

\section{Discussion}

Bileaflet valves are the mechanical valves of choice at the present. Different prosthetic valve models may vary in their propensity for thrombosis, the relative interrelations and proportion of ingrowth tissue and thrombus, the amount of thrombus needed to disturb leaflet motion and the vulnerable zones within the valve.

Data on the pathologic findings in patients with stuck bileaflet valves are sparse. The most extensive data comes from Deviri et al, who studied 112 cases of explanted thrombosed valves, of which $41(36.6 \%)$ were bileaflet. Among these 41 patients, in $30(73.2 \%)$ the thrombus was at the hinge site, causing impairment of both leaflet motion [11]. Vitale et al studied 87 explanted thrombotic prosthetic valves, of which only $12(13.8 \%)$ were bileaflet [1]. It seems that the amount of thrombotic material needed to cause interruption of leaflet motion in bileaflet valves is minimal, especially if it catches the hinge of the valve. A small thrombus may even entrap the hinges of both leaflets $[3,11]$. The lack of disabling embolic events in our patients reflects the careful selection of patients, i.e., the exclusion of patients with large or pedunculated mobile 
thrombi. In addition, our policy is to advocate thrombolysis as the first line of therapy in stuck valve, even in mildly symptomatic or low-risk patients. In contrast, the two largest series (engulfing nearly half of the patients receiving thrombolysis for stuck mitral valve reported in the literature) included patients either on the basis of advanced heart failure or those who were so critically ill that the operative mortality was considered prohibitive $[2,4]$. Above all, we believe that TEE should always be performed before thrombolysis in left-sided valves to exclude large thrombi. In a literature review of 182 left-sided thrombotic episodes, there were 23 episodes of transient or permanent neurologic damage or peripheral emboli (12.6\%) [6]. Fourteen of these episodes were taken from a single study [2] in which TEE was performed only in $11 / 74$ episodes $(14.9 \%)$ : $9 / 41(22.0 \%)$ in stuck mitral valve and $2 / 33(6.1 \%)$ in stuck aortic valve. In the other studies altogether, the above-mentioned complications occurred in $9 / 108(8.3 \%)$, with only two cases of permanent neurologic damage $(1.9 \%)$, one of which occurred when thrombolysis was given, despite the visualization of a mobile thrombus in TEE [12]. We believe that the high rate of cerebral and peripheral emboli reported in the literature reflects poor patient selection, that is, inclusion of patients in whom large left-sided thrombi were not excluded by TEE.

\section{Conclusions}

By comparing data from the main reports on thrombolytic therapy in stuck bileaflet mitral valves, it is evident that the outcome in this study ranks among the best reported in the current literature. In patients with stuck bileaflet mitral valves without large clots, thrombolysis offers a valid alternative to surgery with a high success rate and minimal complications and low mortality. This therapy may be implemented in a wide variety of patients, regardless of symptom duration or severity. Since recurrent episodes may be subclinical, frequent followup echocardiograms are advocated after a successful thrombolysis, and fluoroscopy should be liberally instituted to evaluate leaflet motion. Further studies are required to substantiate the encouraging results of our study and to establish the best thrombolytic regimens.

\section{Acknowledgments}

We are grateful to Dr. Narender Omprakash Bansal, Professor and Head of Department of Cardiology, Grant Medical College and Sir JJ Group of Hospital, Mumbai for allowing us to publish this original article.

\section{Conflict of Interest}

None.

\section{Funding}

None.

\section{References}

1. Vitale N, Renzulli A, Agozzino L, Pollice A, Tedesco N, de Luca Tupputi Schinosa L, Cotrufo M. Obstruction of mechanical mitral prostheses: analysis of pathologic findings. Ann Thorac Surg. 1997;63(4):1101-1106.

2. Roudaut R, Labbe T, Lorient-Roudaut MF, et al. Mechanical cardiac valve thrombosis: Is fibrinolysis justified? Circulation. 1992;86(Suppl II):8-15.

3. Silber H, Khan SS, Matloff JM, Chaux A, DeRobertis M, Gray R. The St. Jude valve. Thrombolysis as the first line of therapy for cardiac valve thrombosis. Circulation. 1993;87(1):30-37.

4. Reddy NK, Padmanabhan TN, Singh S, Kumar DN, Raju PR, Satyanarayana PV, Rao DP, et al. Thrombolysis in left-sided prosthetic valve occlusion: immediate and follow-up results. Ann Thorac Surg. 1994;58(2):462-470; discussion 470-461.

5. Vitale N, Renzulli A, Cerasuolo F, Caruso A, Festa M, de Luca L, Cotrufo M. Prosthetic valve obstruction: thrombolysis versus operation. Ann Thorac Surg. 1994;57(2):365-370.

6. Koller PT, Arom KV. Thrombolytic therapy of left-sided prosthetic valve thrombosis. Chest. 1995;108(6):16831689.

7. Lengyel M, Fuster V, Keltai M, Roudaut R, Schulte HD, Seward JB, Chesebro JH, et al. Guidelines for management of left-sided prosthetic valve thrombosis: a role for thrombolytic therapy. Consensus Conference on Prosthetic Valve Thrombosis. J Am Coll Cardiol. 1997;30(6):1521-1526.

8. Munclinger MJ, Patel JJ, Mitha AS. Thrombolysis of thrombosed St. Jude Medical prosthetic valves: rethrombosis - a sign of tissue ingrowth. J Thorac Cardiovasc Surg. 1998;115(1):248-249.

9. Hurrell DG, Schaff HV, Tajik A. Thrombolytic therapy for obstruction of mechanical prosthetic valves. Mayo Clin Proc. 1996;71(6):605-613.

10. Birdi I, Angelini GD, Bryan AJ. Thrombolytic therapy for left sided prosthetic heart valve thrombosis. J Heart Valve Dis. 1995;4(2):154-159.

11. Deviri E, Sareli P, Wisenbaugh T, Cronje SL. Obstruction of mechanical heart valve prostheses: clinical aspects and surgical management. J Am Coll Cardiol. 1991;17(3):646-650.

12. Dzavik V, Cohen G, Chan KL. Role of transesophageal echocardiography in the diagnosis and management of prosthetic valve thrombosis. J Am Coll Cardiol. 1991;18(7):1829-1833. 